\title{
Vincian Velcro
}

Leonardo demonstrated his innovatory draw ing techniques in his sketch of a human fetus. The w ork was partly inspired by his studies of botany and his dissection of a cow - and it hints at his thoughts on the nature of life itself.

\section{Martin Kemp}

A ll the new graphic modes forged during the Renaissance were realized at the highest level in the drawings of Leonardo da Vinci. Indeed, he invented key techniques, including the exploded view and solid section for the disclosing of natural structures.

His famous drawing of a fetus in the womb is a miracle of intense presentation. The womb, split open like a burst seed-case, reveals the coiled fetus, shaped into compelling roundness by the rhythmic curves of his pen.

The botanical analogy is typical of Leonardo's search for microcosmic parallels between all created forms, and is underscored by the ancillary sketches in the lower centre of the sheet, where successive membranes of the womb are peeled away layer by layer to disclose its tender secrets.

The womb is clearly not that of a human mother. The cotyledonous placenta, composed from separate pads attached across the surface of the womb, has been adapted from the beautiful drawing he made of his dissection of the gravid uterus of a cow. The perfection of design that he found in that dissection could not, in his mind, be other than present in the human womb, complementing the innate sphericality of the chamber in which the new life germinates.

\section{A Iternative configurations for the} interdigitations of the 'male and female parts' of the cotyledons are envisaged in two brilliantly innovatory draw ings to the upper right. The peeling back of one of the pads, separating reluctantly like a Velcro strip, is a graphic device of extraordinary eloquence.

On first glance, the two sketchy diagrams on the sheet, to the centre right and in the bottom right corner, appear to be unrelated - apparently typical of the magpie restlessness of Leonardo's thought. However, the former, which demonstrates how a sphere may be stabilized on an inclined plane by an eccentric weight, probably arose during his meditations on the orientation of the fetus, with its heavy head in its bath of fluid. And the latter, which demonstrates "why a picture seen with one eye will not demonstrate such relief as the object seen with two eyes", serves as a characteristic reminder that even his skills cannot equal the effect of the actual form seen with binocular sight.

But we are still missing the core of Leonardo's vision. In the note below the

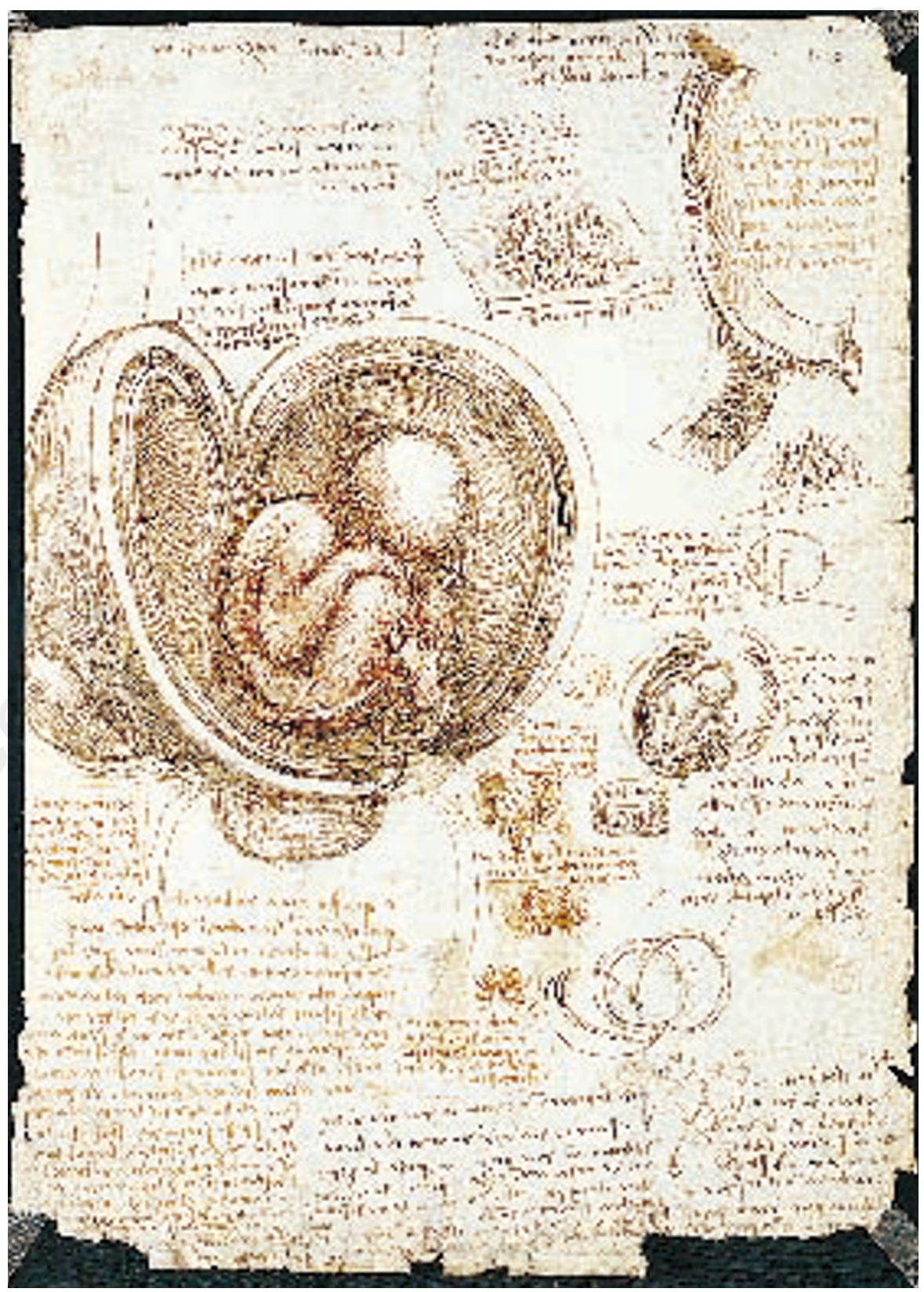

Leonardo da Vinci's “The infant and womb”, in the Royal Library at Windsor Castle.

main drawing, he speculates on a matter that we would not deem to be susceptible to 'anatomical' investigation: "The same soul governs these two bodies, and the desires and fears and sorrows are common to this creature as to all the other animated parts, and from this it arises that something desired by the mother is often found engraved on the limbs of the infant... and a sudden terror destroys the mother and the child."

Viewed in this light, the drawing is a study of what he calls the "great mystery" of the fetus in the waters of the womb, an unawakened life whose thoughts and body are being irresistibly shaped by a oneness of two beings that can never be equalled. There was no such thing as 'descriptive anatomy' for Leonardo, unless we extend description to embrace the profoundest instincts about human life in the greater scheme of things.

Martin Kemp is in the Department of the History of Art, University of Oxford, 35 Beaumont Street, Oxford OX1 2PG, UK.

e-mail:martin.kemp@trinity.oxford.ac.uk 